\title{
SCIENCE, TECHNOLOGY \& KNOWLEDGE
}

\section{5-17 March 2019}

\section{Pile Displacement Measurement Method}

\section{with High Time Resolution for Rapid Loading Test}

\author{
Keita Nishio, Takashi Kaburagi, Kaoru Kuramoto, \\ Toshiyuki Matsumoto, Satoshi Kumagai and Yosuke Kurihara
}

Aoyama Gakuin University

\begin{abstract}
In the building industry, a rapid loading test is used to examine the bearing capacity of a pile, wherein a mass is dropped on the pile from above and it is displaced for 0.05 to $0.20 \mathrm{~s}$. Two parameters are evaluated by this test: load applied on the pile head and displacement of the pile. Owing to the short loading time, a displacement measurement with high time-resolution is necessary, which is achieved with a high-speed camera. In this study, we propose a measurement method for pile displacement with a line scan camera that has higher timeresolution than a high-speed camera. This method is consisted of an illuminant, a curved mirror, a screen and a line scan camera. A laser beam from an illuminant is incident on a mirror attached to the side of the pile; the mirror reflects the light to a screen, which is then tracked by the line scan camera to an output image. Binarization and curve detection are applied to this image to estimate pile displacement. Further, to remove noise from the curve detection output, we constructed an equation for motion of the pile during the test and devised a Kalman filter. To verify the proposed method, a scaled miniature pile was built for the rapid loading test, and the absolute error between the estimated displacement and true displacement measured using an eddy current displacement meter was evaluated. The average absolute error was $0.52 \pm 0.18$ $\mathrm{mm}$.
\end{abstract}

Keywords: bearing capacity; Kalman filter; laser reflection; line scan camera; optical lever 